\title{
Tibiakopf - Klassifikation und Prinzipien der Behandlung
}

Jan Friederichs, Fabian Stuby

\section{Einleitung}

Verletzungen des Tibiakopfes entstehen in den meisten Fällen durch eine hochgradige Krafteinwirkung auf das Kniegelenk, wie es bei Verkehrsunfällen, Stürzen oder Sportverletzungen (z.B. alpines Skifahren) vorkommt. Da es hierbei oft zu einer schweren Zertrümmerung der Gelenkfläche kommt, ist der Krankheitsverlauf langwierig und das funktionelle Ergebnis nicht immer zufriedenstellend. Schmerzen, Beweglichkeitseinschränkungen, Achsfehlstellungen und frühzeitiger Gelenkverschleiß können die Folge sein. Daher ist die Wiederherstellung der Gelenkkongruenz das primäre Ziel. Hierfür stehen dem Operateur moderne Operationstechniken unter Verwendung von anatomischen winkelstabilen Implantaten zur Verfügung. Ein besonderes Augenmerk muss jedoch auf passende, weichteilschonende Zugangswege gerichtet werden.

Wichtig erscheint hierbei die klinische Untersuchung, die auch die Erhebung des Gefäßstatus und des neurologischen Status beinhaltet. Neben dem Ausschluss eines akuten oder drohenden Kompartmentsyndroms muss eine genaue Beurteilung der Weichteile erfolgen, eventuelle Begleitverletzungen müssen ausgeschlossen werden. Der konventionell radiologischen Diagnostik schließt sich die computertomografische Diagnostik mit 3-D-Rekonstruktion an, dies ist zwingend, da nur so eine ausreichende Frakturanalyse und -klassifikation durchgeführt werden kann. Bei Luxationsfrakturen oder speziellen Fragestellungen kann auch eine Ergänzung der Diagnostik durch Angio-CT oder MRT erforderlich werden. Die Erstbehandlung erfolgt bei Kniegelenkluxationsfrakturen, instabilen Frakturen, kritischen Weichteilen oder Kompartmentsyndrom durch Stabilisierung mittels gelenküberbrückendem Fixateur externe (plus Kompartmentspaltung), bei einfachen, stabilen Frakturen mit unkritischen Weichteilen durch Ruhigstellung im Kletttutor. Die Analyse der Fraktur mittels Computertomografie kann dann im Anschluss daran durchgeführt werden („span it, scan it, fix it") und die definitive Stabilisierung nach sorgfältiger Operationsplanung im Intervall nach 4-10 Tagen durchgeführt werden.

\section{Klassifikation}

Klassifikationen von Tibiakopffrakturen wurden in den vergangenen Jahrzehnten mit dem Ziel entwickelt, eine optimale Behandlungsstrategie abzuleiten und dadurch letztlich ein besseres Behandlungsergebnis erzielen zu können. Die weltweit am häufigsten angewendete Schatzker-Klassifikation [1] und die im deutschsprachigen Raum weit verbreitete AO-Klassifikation (AO: Arbeitsgemeinschaft Osteosynthese) [2] sind umfangreich untersucht und beschrieben worden und gelten als Standardklassifikationen für proximale Tibiafrakturen. Gleiches gilt für die Einteilung der Tibiakopfluxationsfrakturen nach Moore et al. [3]. Als bildgebende Verfahren dieser Klassifikationen dient das konventionelle Röntgen (anterior-posteriore Röntgenprojektion). Neben der geringen Intra- und Interbeobachterreliabilität ist zu beachten ist, dass insbesondere bei komplexen Brüchen nur wenige Frakturmuster abgebildet sind [4, 5]. Für die sorgfältige Analyse der Fraktur und Planung der Osteosynthese haben diese Klassifikationen daher, insbesondere bei komplexen Frakturmustern, nur noch einen historischen Wert.

Auch die Modifikationen der oben beschriebenen „klassischen" Klassifikationssysteme erbrachten keine richtungsweisende Verbesserung [6]. Erst durch die Einführung der CT-Bildgebung inklusive 3-D-Rekonstruktionen konnten Fortschritte in der Planung des operativen Vorgehens und damit im Zusammentreffen mit der Weiterentwicklung der Implantate (anatomisch, winkelstabil) auch eine Verbesserung des funktionellen Ergebnisses erzielt werden. Als Beispiel werden in $>$ Abb. 1 eine konventionelle Aufnahme einer Tibiakopfluxationsfraktur im Vergleich mit repräsentativen Schnitten der gleichen Fraktur in der Computertomografie gezeigt. Als Folge wurden in den letzten Jahren Klassifikationen entwickelt, die auf der Analyse der Computertomografie basieren und aus denen Therapiealgorithmen abzuleiten sind. Dies hat sich insbesondere bei komplexen, mehrfragmentären Frakturen in der klinischen Praxis bewährt. Als Beispiele sind hier die 3-Säulen-Klassifikation nach Luo et al. zu nennen [7], im Jahr 2016 wurde von der Arbeitsgruppe um K.-H. Frosch eine 10-Säulen-Klassifikation vorgeschlagen. Die Studie betont die Wichtigkeit der Analyse der Frakturlinien, insbesondere in den hinteren Segmenten des Tibiakopfes bei komplexen Tibiafrakturen, teilt diese 

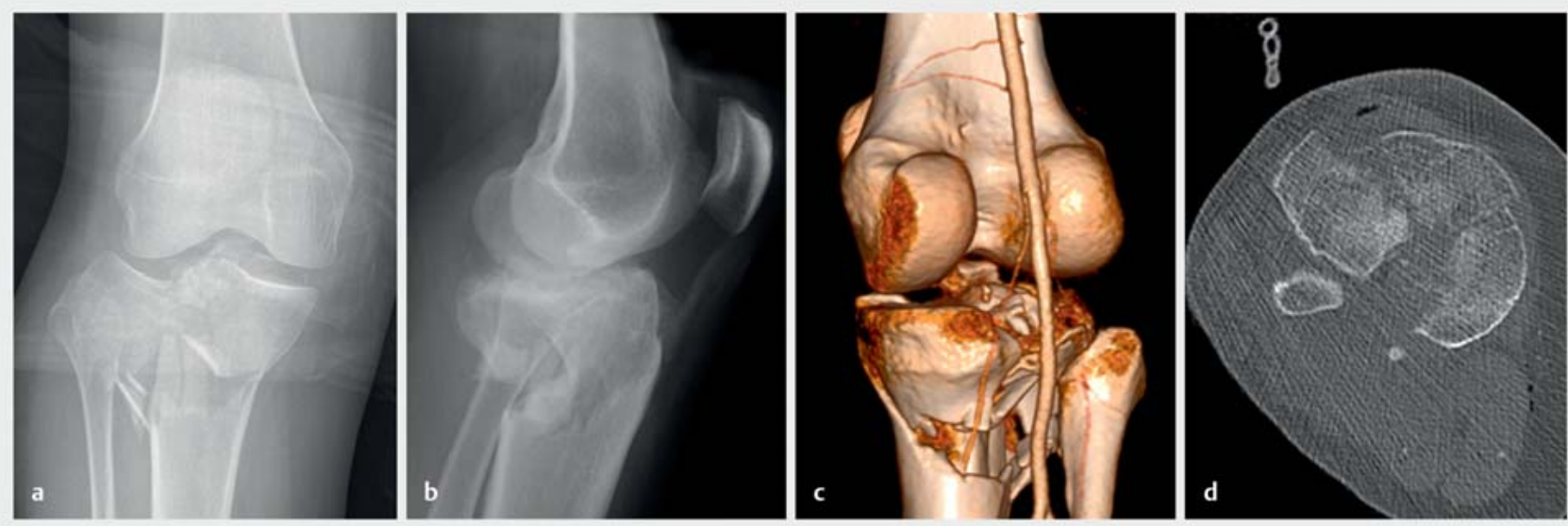

- Abb. 1 Darstellung einer komplexen proximalen Tibialuxationsfraktur im konventionellen Röntgen und in der (3-D)-Computertomografie. Eine eingehende Frakturanalyse ist in der konventionellen Röntgendiagnostik nicht möglich.
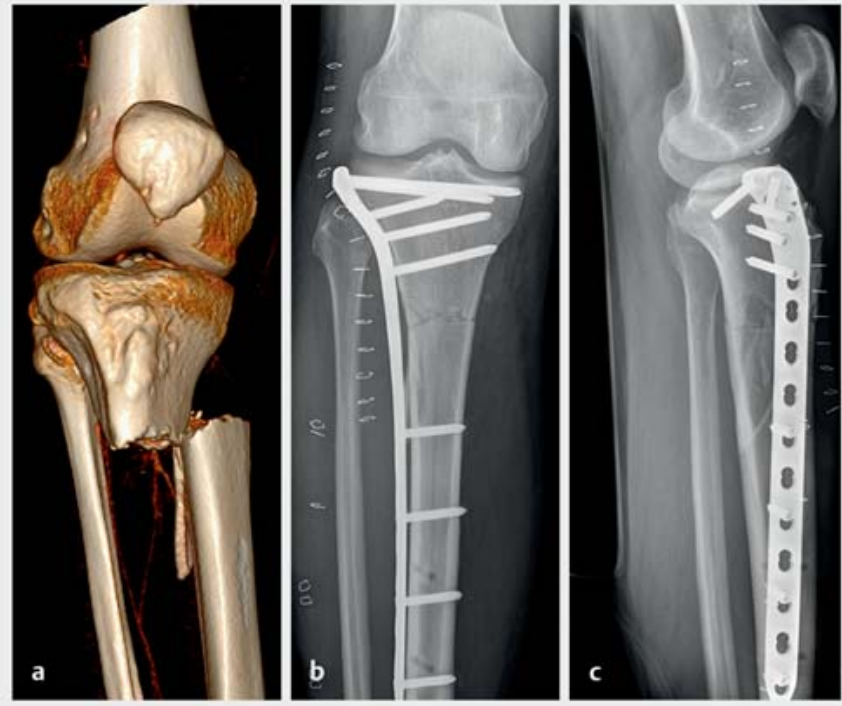

- Abb. 2 Extraartikuläre Fraktur der proximalen Tibia nach Hochrasanztrauma in der 3-D-Computertomografie (a). Operative Stabilisierung mit eingeschobener winkelstabiler Platte (b, c).

ein und schlägt ein Therapiekonzept vor, das die Verwendung von hinteren Zugangswegen impliziert [8]. Die hohe Bedeutung der exakten Reposition und Fixierung der dorsalen Anteile ist entscheidend für das Langzeitergebnis. Einen ähnlichen Ansatz wählten Pätzold et al. in ihrer 2017 veröffentlichten Studie [9]. Hierin werden Typ-C-Frakturen in 3 Typen eingeteilt, die sich am Vorhandensein und Verlauf der koronaren Frakturlinie in den axialen CT-Schichten orientieren. Die Klassifikation besitzt eine hervorragende Intra- und Interbeobachterreliabilität und erlaubt eine Therapieentscheidungshilfe bez. des Zugangsweges. Auch hier spielt die Anwendung von dorsalen Zugängen eine große Rolle.

\section{Prinzipien der Osteosynthese}

Die konservative Therapie ist beim Erwachsenen selten indiziert, dies nur bei stabilen, nicht dislozierten Frakturen und/oder patienteneigenen Kontraindikationen. Wie oben beschrieben, erfolgt die Initialbehandlung durch Ruhigstellung im Strecktutor oder durch Stabilisierung mittels Fixateur externe. In unseren Breitengraden ist die Ausbehandlung im Fixateur externe oder im Ringfixateur eher eine Seltenheit und nur bei desolaten Weichteilverhältnissen oder Infektverläufen sowie seltenen individuellen Indikationen zu empfehlen.

Zu empfehlen ist eine definitive Stabilisierung im Intervall (4-10 Tage), die Initialversorgung besteht in der Ruhigstellung in der Schiene oder mittels Fixateur externe.

\section{„Einfache Frakturen“}

Unter „einfachen Frakturen“ sollen hier kurz die möglichen Behandlungsweisen von extraartikulären proximalen Tibiafrakturen (AO-Typ A) und reinen Spaltbrüchen (AO-Typ B) erläutert werden. Die klassische Methode der Stabilisierung von extraartikulären Frakturen der proximalen Tibia stellt die winkelstabile Plattenosteosynthese dar. Diese erfolgt bestenfalls, wie in $\mathbf{A} \mathbf{A b} \mathbf{b} . \mathbf{2}$ dargestellt, als eingeschobene Platte, so konnten die in der Vergangenheit beschriebenen hohen Infektraten [10] auf ein akzeptables Maß reduziert werden. Aufgrund der Winkelstabilität der neuen anatomischen Implantate bietet die laterale Plattenlage hier meist eine ausreichende Stabilität. Entscheidend sind die anatomische Reposition zur Gewährleistung einer hohen intrinsischen Stabilität, eine korrekte Achsausrichtung und ein weichteilschonendes 
Arbeiten. Intramedulläre Lastträger mit veränderter Geometrie und veränderten proximalen Verriegelungsoptionen sowie die Verwendung von additiven Platten können in bestimmten Fällen eine sinnvolle Therapieoption sein, stellen bei uns aber eher die Ausnahme dar.

Der reine Spaltbruch des lateralen Tibiaplateaus rechtfertigt die alleinige Anwendung von Schrauben. Wie in - Abb. 3 gezeigt, kann die nicht dislozierte oder gering dislozierte Fraktur minimalinvasiv reponiert und gedeckt mittels kanülierter Schrauben stabilisiert werden, wobei eine exakt parallele und gelenknahe Ausrichtung der Schrauben ohne medialen Überstand gefordert werden muss. Gegebenenfalls kann zur Kontrolle der exakten, stufenfreien Reposition der Gelenkfläche die Arthroskopie zur Hilfe genommen werden, der Übertritt der Spülflüssigkeit über die Frakturspalten mit Schwellungen bis hin zum Kompartmentsyndrom [11] kann durch eine kurze Arthroskopiezeit mit geringen Drücken verhindert werden. Ein Beispiel ist in > Abb. $\mathbf{4}$ dargestellt.

\section{Impressionsfrakturen des lateralen Tibiaplateaus}

Impressionsfrakturen (Typ B2) des lateralen Tibiaplateaus sind eine Domäne der Plattenosteosynthese über den anterolateralen Standardzugang. Nach Darstellung der Fraktur, lateraler Arthrotomie mit Reposition und Anschlingen des möglicherweise abgerissenen Außenmeniskus wird entweder über die Fraktur oder über ein distal angelegtes Knochenfenster unter Sicht bzw. Durchleuchtungskontrolle die Gelenkfläche durch Hochstößeln imprimierter Fragmente rekonstruiert und temporär mit Kirschner-Drähten fixiert. In vielen Fällen ist zur besseren Abstützung die Unterfütterung mit Beckenkammspan, Eigen- oder Fremdspongiosa oder Knochenersatzmaterialien erforderlich. Die Stabilisierung wird dann mit einem winkelstabilen anatomischen Implantat durchgeführt, in Abhängigkeit von der Frakturmorphologie und der Anatomie als Kleinfragment- oder Großfragmentplatte. Eine zusätzliche Stabilität kann durch die Gitterstruktur (JailTechnik) von 2 in anteroposteriorer Richtung eingebrachten Einzelschrauben erreicht werden ( $\bullet$ Abb. 5). Eine ausreichende Anhebung des Plateaus ohne Verbreiterung mit stufenfreier Gelenkfläche ist hier das Operationsziel.

\section{Tibiakopfluxationsbrüche}

Die Verrenkungsbrüche (Typ-C-Frakturen) gehen oft mit einer nicht unerheblichen Weichteil- und Bandverletzung einher. Offene Frakturen, ein manifestes oder drohendes Kompartmentsyndrom und ein Gefäß- oder Nervenschaden (N. fibularis) kommen hier häufig vor und müssen präoperativ abgeklärt werden. Da hier ein zweizeitiges Vorgehen (Fixateur externe initial und die definitive Versorgung nach 4-10 Tagen) empfohlen wird, bleibt genug Zeit zur genauen Analyse der Fraktur und Operationsplanung (Zeitpunkt, Zugangsweg, Weichteile, Reposition, Implantate, Implantatlage). Weitere Verletzungsbestand-

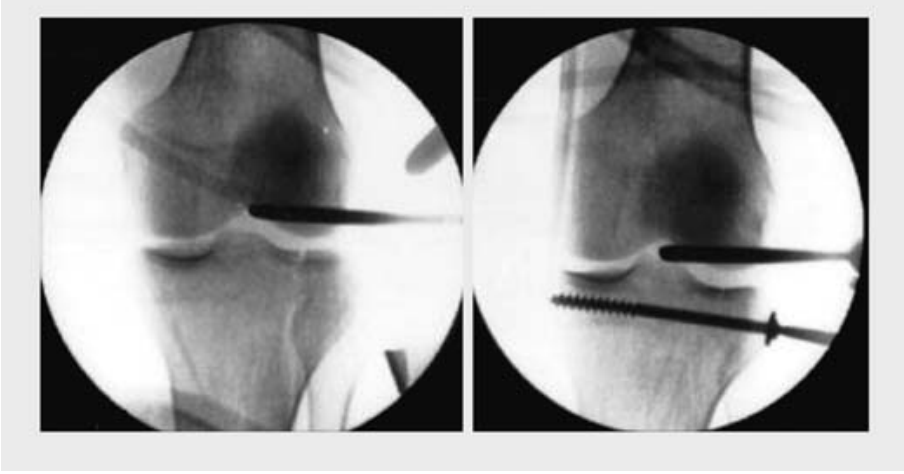

- Abb. 3 Spaltbruch des lateralen Tibiaplateaus (Typ B1) und operativer Stabilisierung durch gedeckte Verschraubung. Man beachte das einliegende Arthroskop sowie den Verschluss des Frakturspaltes nach Schraubenosteosynthese.

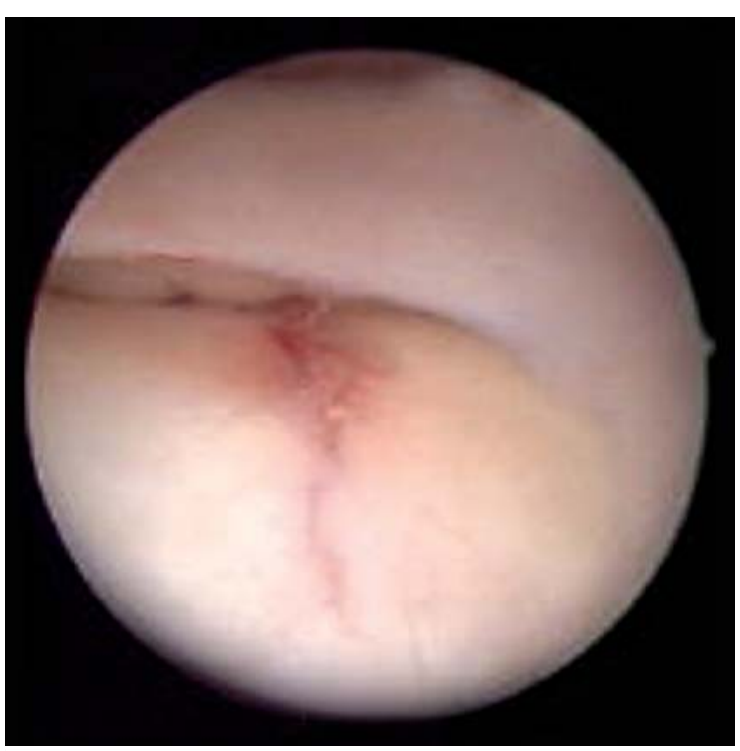

- Abb. 4 Intraoperativer arthroskopischer Befund mit stufenfreiem Verschluss des Frakturspaltes.

teile wie knöcherne Kreuzbandausrisse, knöcherne Kollateralbandausrisse, zu erwartende Meniskusabrisse und freie Knorpelfragmente müssen bedacht werden, es ist wichtig, die Fraktur „zu verstehen“. Hier ist das mediale Schlüsselfragment ( $\boldsymbol{A}$ Abb. 6 ) von besonderer Bedeutung, da nur über dieses eine korrekte Reposition gelingt.

\section{Merke}

Die Analyse der Fraktur beinhaltet auch eine Repositionstaktik und die Planung des passenden Implantats über den passenden Zugangsweg.

Die Reposition kann bei komplexen, mehrfragmentären Frakturen aus 2 Taktiken bestehen, zum einen kann versucht werden, zunächst die Gelenkfläche zu rekonstruie- 


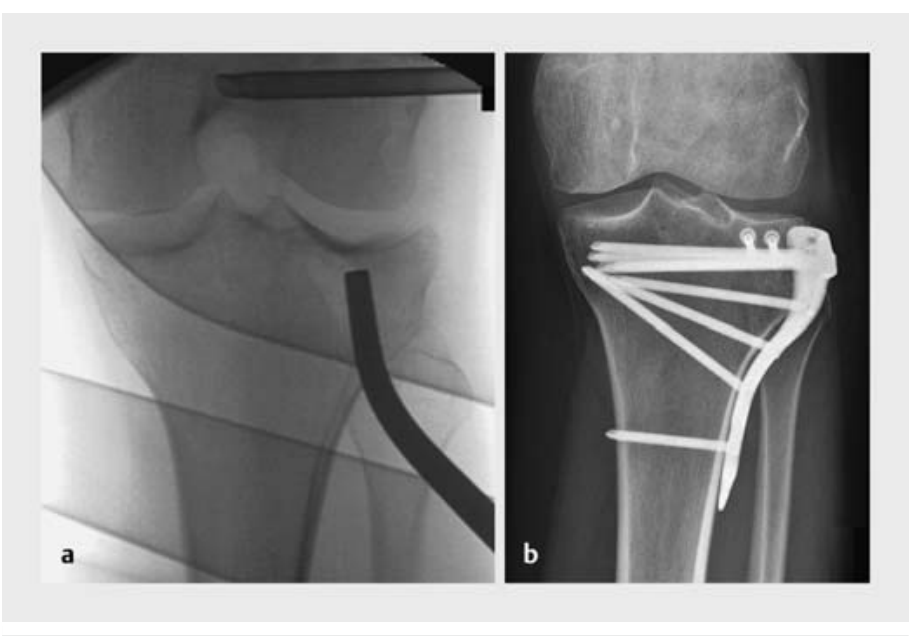

- Abb. 5 Hochstößeln des lateralen Tibiaplateaus unter Bildwandlerkontrolle (a). In b wird ein Beispiel der anschließenden Osteosynthese mit winkelstabiler anatomischer Kleinfragmentplatte und 2 in anteroposteriorer Richtung eingebrachten kanülierten Schrauben gezeigt.
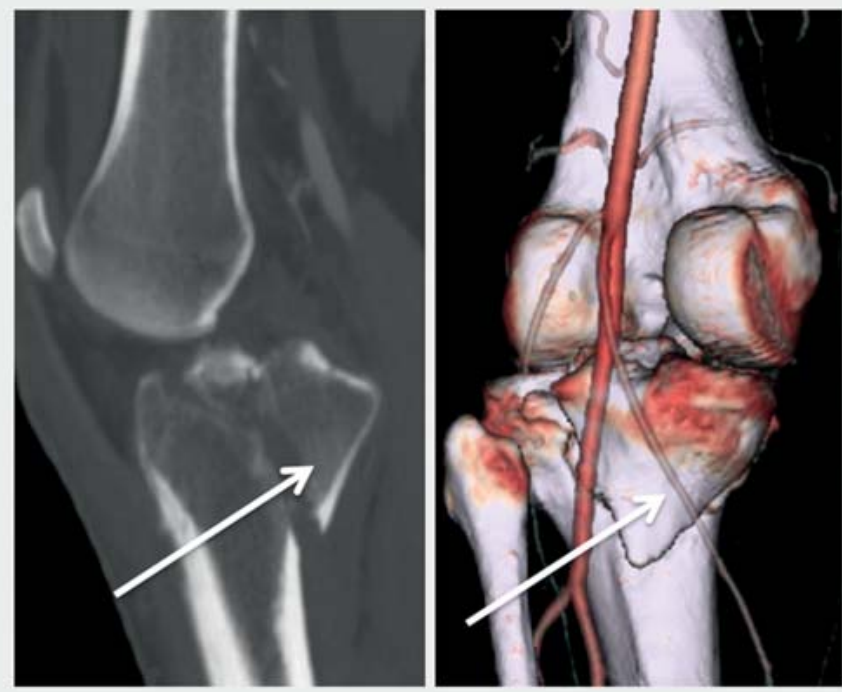

Abb. 6 Das mediale Schlüsselfragment bei Luxationsfrakturen der proximalen Tibia (Typ C).

ren („aus C mach $A$ “) und diese dann wie eine extraartikuläre Fraktur mit dem Schaft zu verbinden. Dies gelingt am besten unter Verwendung von Einzelschrauben in Kombination mit einer lateralen winkelstabilen Platte. Häufiger führen wir die Reposition in anderer Reihenfolge nach dem Prinzip „aus C mach B“ durch, hierbei erfolgt die Reposition des Schaftfragmentes an das mediale Schlüsselfragment, die Rekonstruktion des lateralen Plateaus wird dann wie bei lateralen Impressionsfrakturen durchgeführt.

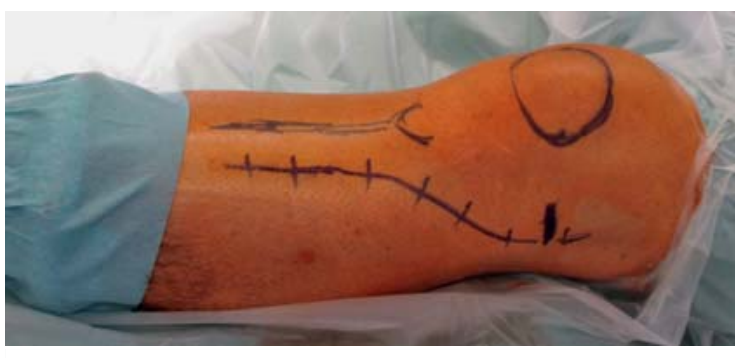

- Abb. 7 Anterolateraler Standardzugang zur proximalen Tibia. Wir verwenden eine geschwungene Schnittführung über das Tuberculum gerdyi, auch eine gerade Schnittführung ist möglich. Eingezeichnet sind die Tuberositas tibiae, die Tibiavorderkante sowie die Patella.

\section{Zugangswege}

Nach sorgfältiger Analyse der Fraktur wird der optimale Zugangsweg gewählt, wobei hier auch die Weichteilsituation in die Überlegung einbezogen werden muss. In den meisten Fällen sind bei komplexen Luxationsfrakturen 2 Zugänge erforderlich, um die proximale Tibia mittels Doppelplattenosteosynthese zu stabilisieren. Der am häufigsten verwendete Zugang ist der anterolaterale Standardzugang, über den eine Rekonstruktion des lateralen Tibiaplateaus durchgeführt werden kann. Bei uns wird die geschwungene Schnittführung über das Tuberculum gerdyi gewählt ( $\triangleright$ Abb. 7), möglich ist auch eine gerade Schnittführung [12]. Weichteilschonend wird nun der Tractus iliotibialis inzidiert und vom Tuberculum gerdyi nach ventral und dorsal abpräpariert. Die Darstellung der Fraktur erfolgt streng subperiostal, die Arthrotomie horizontal unterhalb des Außenmeniskus. Über diesen Zugang kann das anterolaterale Kompartiment des Gelenkes eingesehen werden, die Rekonstruktion und Anhebung des lateralen Plateaus mit Unterfütterung und Stabilisierung sind möglich, der Außenmeniskus kann refixiert werden ( $\bullet$ Abb. 8 ).

Ein posterolateraler Zugang wird bei uns nur selten durchgeführt. Unterschiedliche Methoden sind beschrieben (mit oder ohne Fibulaosteotomie, modifiziert anterolateral, erweiterter lateraler Zugang, direkt über posterior), als sehr weichteilschonend gilt der von Frosch beschriebene posterolaterale Zugang, der über eine Hautinzision direkt über dem Fibulakopf über 2 Fenster ohne Osteotomie der Fibula und mit Darstellung des Fibularisnervs eine gute Übersicht in den postero- und anterolateralen Quadranten ermöglicht [12].

Merke

Bei der Wahl des Zugangsweges muss neben dem Frakturtyp auch der Weichteilstatus berücksichtigt werden.

Bei uns wird bei bikondylären Frakturen meist mit einem posteromedialen Zugang (nach Lobenhoffer et al. [13] 


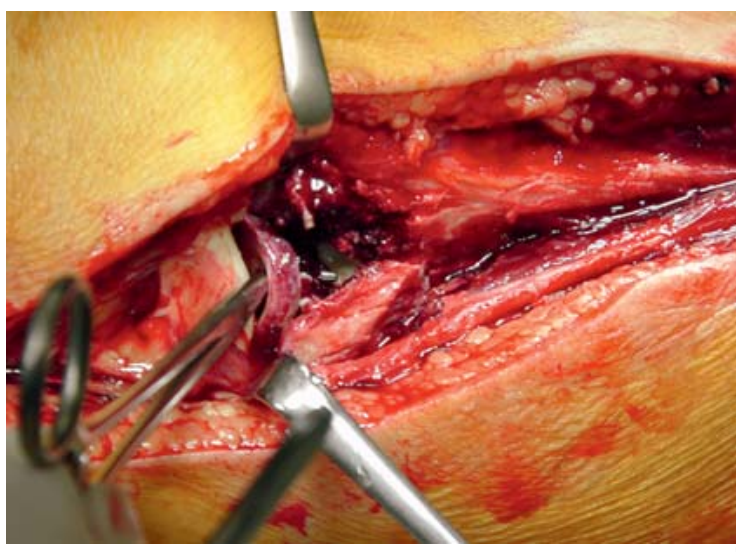

- Abb. 8 Darstellung des abgerissenen Außenmeniskus (Klemme) nach querer Arthrotomie. Der Meniskus wird angeschlungen und nach Abschluss der Reposition und Osteosynthese refixiert.

begonnen, um, wie oben beschrieben, zunächst die Reposition des medialen Schlüsselfragmentes an den Schaft und damit die Rekonstruktion der medialen Säule zu erreichen. Bei leicht angewinkeltem Knie wird hier der Hautschnitt entlang der posterioren Tibiakante geführt ( A Abb. 9), nach Darstellung der Hamstring-Sehnen kann der extraartikuläre Frakturverlauf meist unter Sicht anatomisch reponiert werden. Es stehen winkelstabile anatomische Implantate zur Verfügung wobei verhindert werden muss, durch Fixierung von Fragmenten die spätere Reposition der lateralen Fragmente (über einen separaten anterolateralen Zugang) zu behindern. Dies gelingt durch Verwendung von kürzeren, monokortikalen Schrauben. Die vollständige Stabilisierung gelingt so ohne Umlagerung des Patienten, Einzelschrauben können die Plat-

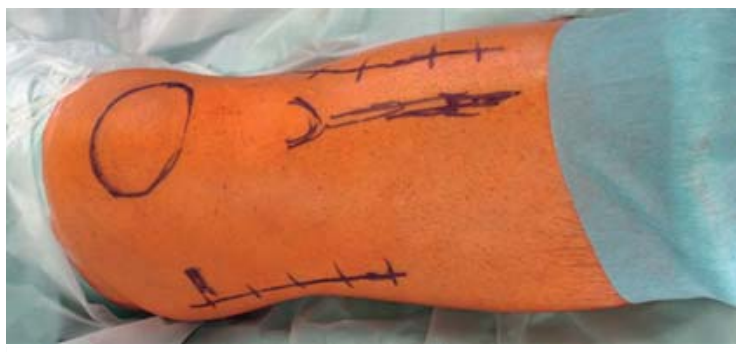

- Abb. 9 Posteromedialer Zugang, hier in Kombination mit dem anterolateralen Standardzugang zur operativen Stabilisierung von bikondylären Tibiakopffrakturen. Eingezeichnet sind neben dem Zugang die Tuberositas tibiae, die Tibiavorderkante sowie die Patella.

tenosteosynthesen sinnvoll unterstützen und können Einzelfragmente (z.B. Tuberositas tibiae) stabil refixieren. Der früher häufig verwendete sehr komplikationsbehaftete anteriore Zugang wird bei uns aufgrund der ausgedehnten Ablösung der Weichteile nicht mehr durchgeführt.

Ist aufgrund der notwendigen Fragmentfixierung (mehrfragmentär, großes dorsales Gelenkfragment) in den posterioren Quadranten eine ausreichende Darstellung über den posteromedialen Zugang nicht möglich, so ist ein direkter posteromedialer Zugang in Bauchlage über eine Schnittführung in der Kniekehle ein optimaler Zugangsweg [14-16]. Über den Zugang nach Trickey, der allerdings nur noch sehr selten verwendet wird und weitgehend vom dorsomedialen limitiert invasiven Zugang nach Frosch abgelöst wurde, können die dorsalen Anteile des Tibiakopfes sehr gut dargestellt werden. Hier wird über einen geraden, über dem medialen Gastroknemius-
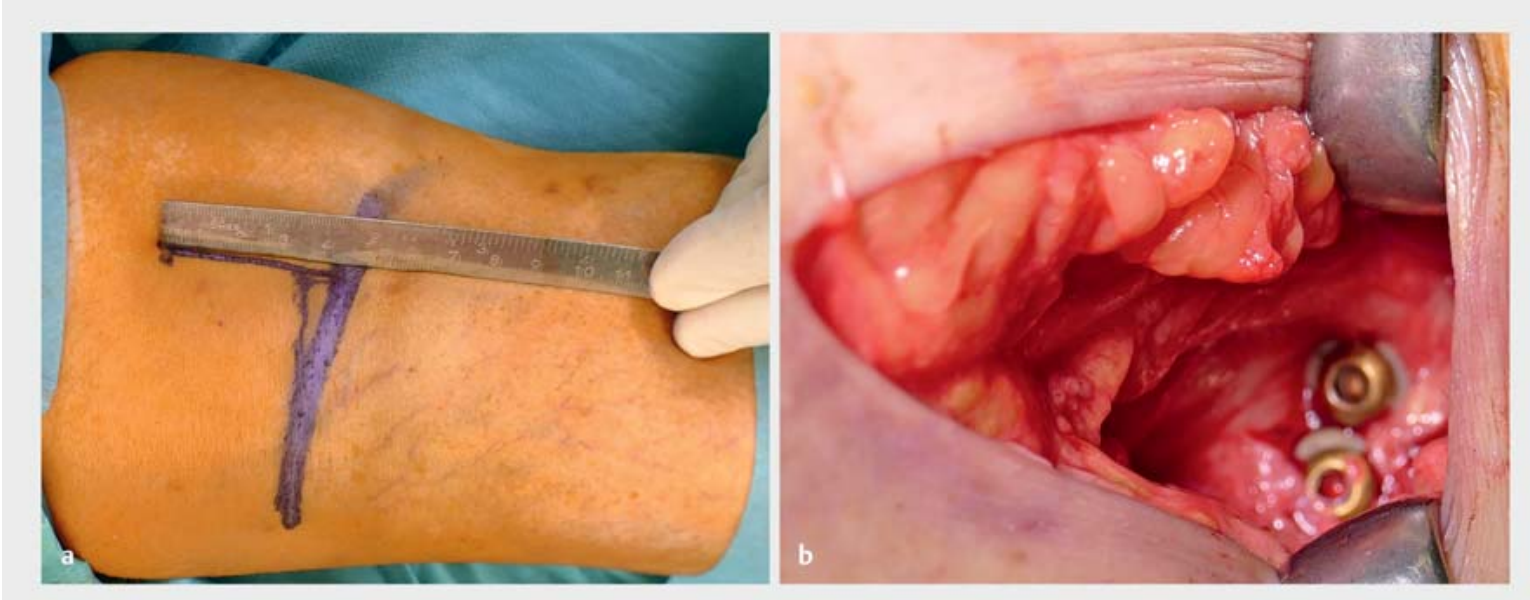

- Abb. 10 Dorsomedial limitiert invasiver Zugang nach Frosch als Beispiel für einen posterioren Zugang zur proximalen Tibia in Bauchlage (a). In Abhängigkeit von der Verletzung (knöcherner Ausriss hinteres Kreuzband) kann dieser, wie in (b) dargestellt, auch rein proximal der Beugeseite liegen. Allerdings darf er bei Erweiterung des Hautschnittes die Beugefalte nicht kreuzen. (Quelle: PD Dr. Steffen Schröter, BG Klinik Tübingen). 


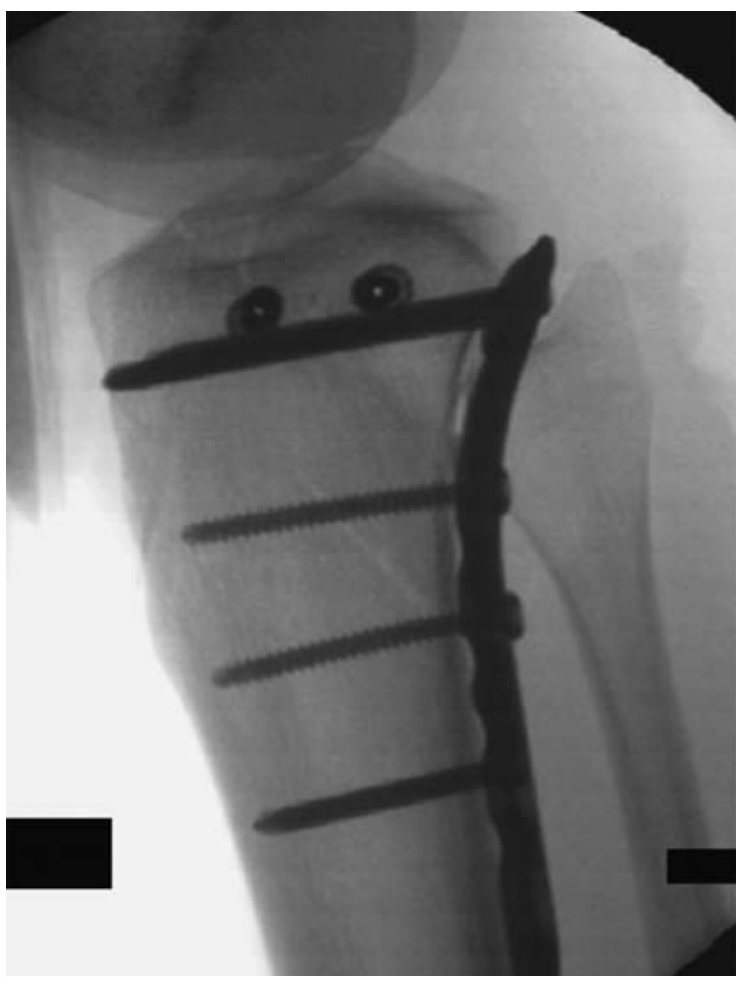

Abb. 11 Beispiel einer dorsalen Abstützplatte eingebracht über den dorsalen Zugang in Bauchlage. Zusätzlich wurden 2 Einzelschrauben zur Unterstützung des Plateaus von lateral eingebracht.

kopf geführten Hautschnitt der gesamte Gastroknemius nach lateral gehalten. Unter Schonung des Gefäß-NervenBündels kann so die hintere Kapsel dargestellt werden ( Abb. 10).

\section{Merke}

CAVE: Der Hautschnitt darf die Beugefalte nicht kreuzen, da aufgrund von Narbenkontrakturen eine Bewegungseinschränkung entstehen kann.

Eine Ablösung oder Einkerbung der Gastroknemiusansätze ist in fast allen Fällen nicht erforderlich, je nach Länge der Hautinzision kann sowohl das posteromediale als auch das posterolaterozentrale Tibiaplateau erreicht werden. Neben Einzelschrauben ist hier die abstützende Platte das Implantat der Wahl ( $\triangleright$ Abb. 11), auch hier muss die Implantatlage/Schraubenlänge die Kombination mit einem 2. Zugang/Implantat antizipieren.

\section{Kernaussagen}

Bei komplexen Frakturen des Tibiakopfes ist eine eingehende Analyse des Frakturtyps und der Frakturlinien in der Computertomografie erforderlich. In der initialen Diagnostik müssen das Kompartmentsyndrom, Gefäßund Nervenschäden sowie Begleitverletzungen ausgeschlossen werden, fast immer ist ein zweizeitiges Vor- gehen erforderlich. Der Weichteilstatus spielt eine bedeutsame Rolle und muss in die spätere definitive Stabilisierung einbezogen werden. Eine Vielzahl an Zugangswegen und Implantatlagen steht zur definitiven Stabilisierung zur Verfügung.

Interessenkonflikt

Die Autoren geben an, dass kein Interessenskonflikt besteht.

Autorinnen/Autoren

Jan Friederichs

Prof. Dr., Leitender Oberarzt, BG Unfallklinik Murnau

Fabian Stuby

PD Dr., Ärztlicher Direktor, BG Unfallklinik Murnau

\section{Korrespondenzadresse}

\author{
Prof. Dr. Jan Friederichs \\ BG Unfallklinik Murnau \\ Professor-Küntscher-Str. 8 \\ 82418 Murnau \\ Tel.: $08841 / 48-4731$ \\ Fax: $08841 / 48-2203$ \\ Jan.Friederichs@bgu-murnau.de
}

Literatur

[1] Schatzker J, McBroom R, Bruce D. The tibial plateau fracture. The Toronto experience 1968-1975. Clin Orthop Relat Res 1979; (138): 94-104

[2] Müller ME. The comprehensive Classification of Fractures of long Bones. Berlin: Springer; 1990

[3] Moore TM, Patzakis M], Harvey JP. Tibial plateau fractures: definition, demographics, treatment rationale, and long-term results of closed traction management or operative reduction. J Orthop Trauma 1987; 1: 97-119

[4] Brunner A, Horisberger M, Ulmar B et al. Classification systems for tibial plateau fractures; does computed tomography scanning improve their reliability? Injury 2010; 41: 173-178

[5] Doornberg JN, Rademakers MV, Van den Bekerom MP et al. Two-dimensional and three-dimenisional computed tomography for the classification and characterization of tibial plateau fractures. Injury 2011; 42: 1416-1425

[6] Millar SC, Arnold JB, Thewlis D et al. A systematic literature review of tibial plateau fractures: what classifications are used and how reliable and useful are they? Injury 2018; 49: 473490

[7] Luo CF, Sun H, Zhang B et al. Three-column fixation of complex tibial plateau fractures. J Orthop Trauma 2010; 24: 683-692

[8] Krause M, Preiss A, Müller $G$ et al. Intra-articular tibial plateau fracture characteristics according to the "Ten segment classification“. Injury 2016; 47: 2551-2557

[9] Pätzold R, Friederichs ], von Rüden C et al. The pivotal role of the coronal fracture line for a new three-dimensional CTbased fracture classification of bicondylar proximal tibial fractures. Injury 2017; 48: 2214-2220 
[10] Young M], Barrack RL. Complications of internal fixation of tibial plateau fractures. Orthop Rev 1994; 23: 149-154

[11] Petersen W, Zantop T, Raschke M. Tibiakopffraktur. Unfallchirurg 2006; 109: 235-244

[12] Krause M, Müller G, Frosch KH. Chirurgische Zugänge bei Tibiakopffrakturen. Unfallchirurg 2018; 121: 569-582

[13] Lobenhoffer P, Gerich T, Bertram T et al. [Particular posteromedial and posteroateral approaches for the treatment of tibial head fractures]. Unfallchirurg 1997; 100: 957-967

[14] Galla M, Lobenhoffer P. Der direkte dorsale Zugangsweg zur Versorgung posteromedialer Luxationsfrakturen des Tibiakopfes. Unfallchirurg 2003; 106: 241-247
[15] Muhm M, Schneider P, Ruffing T et al. Posterozentraler Zugang zum dorsalen Tibiakopf. Unfallchirurg 2014; 117: 813-821

[16] Friederichs J, Trapp O, Stuby F et al. Prinzipien der Osteosynthese an der proximalen Tibia. Trauma Berufskrankh 2018. doi:10.1007/s10039-018-0392-9

\section{Bibliografie}

DOI https://doi.org/10.1055/a-0765-8340

Online-publiziert 15.01.2019 | OP-JOURNAL 2019; 35: 93-99

(c) Georg Thieme Verlag KG Stuttgart · New York ISSN 0178-1715 